\title{
Modeling and removal of optical ghosts in the PROBA-3/ASPIICS externally occulted solar coronagraph
}

\author{
S. V. Shestov ${ }^{1,2}$, A. N. Zhukov ${ }^{1,3}$, and D. B. Seaton ${ }^{4,5}$ \\ ${ }^{1}$ Solar-Terrestrial Centre of Excellence - SIDC, Royal Observatory of Belgium, Avenue Circulaire 3, 1180 Brussels, Belgium \\ e-mail: s.shestov@oma.be \\ 2 Lebedev Physical Institute, Leninskii prospekt, 53, 119991 Moscow, Russia \\ 3 Skobeltsyn Institute of Nuclear Physics, Moscow State University, Leninskie gory, 119991 Moscow, Russia \\ ${ }_{5}^{4}$ Cooperative Institute for Research in Environmental Sciences, University of Colorado at Boulder, Boulder, CO 80305, USA \\ 5 National Centers for Environmental Information, National Oceanic and Atmospheric Administration, Boulder, CO 80305, USA
}

Received 6 November 2018 / Accepted 8 December 2018

\begin{abstract}
Context. ASPIICS is a novel externally occulted solar coronagraph, which will be launched onboard the PROBA-3 mission of the European Space Agency. The external occulter will be placed on the first satellite $\sim 150 \mathrm{~m}$ ahead of the second satellite that will carry an optical instrument. For $6 \mathrm{~h}$ per orbit, the satellites will fly in a precise formation, constituting a giant externally occulted coronagraph. The large distance between the external occulter and the primary objective will allow observations of the white-light solar corona starting from extremely low heights $\sim 1.1 R_{\odot}$.

Aims. We aim to analyze the possible influence of optical ghost images formed inside the telescope and to develop an algorithm for their removal.

Methods. We implement the optical layout of ASPIICS in Zemax and study the ghost behavior in sequential and nonsequential regimes. We identify sources of the ghost contributions and analyze their geometrical behavior. Finally we develop a mathematical model and software to calculate ghost images for any given input image.

Results. We show that ghost light can be important in the outer part of the field of view, where the coronal signal is weak, since the energy of bright inner corona is redistributed to the outer corona. However, the model allows for the ghost contribution to be removed. Due to the large distance between the external occulter and the primary objective, the primary objective does not produce a significant ghost. The use of the Lyot spot in ASPIICS is not necessary.
\end{abstract}

Key words. Sun: corona - instrumentation: high angular resolution - telescopes - methods: numerical

\section{Introduction}

The difficulty of observing the low solar corona in white-light coronagraphic observations is caused by two factors: the very high dynamic range of the corona, and the presence of intense light from the solar disk diffracted by the external occulter (EO; Koutchmy 1988; Shestov \& Zhukov 2018).

The high dynamic range of coronal brightness, which may vary from $10^{-5}$ of mean solar brightness (MSB) at $1.01 R_{\odot}$ to less than $10^{-10} \mathrm{MSB}$ at $3 R_{\odot}$, makes instrumentation intended to observe this region in visible light very sensitive to the presence of stray light of any nature, like diffraction, scattering, or ghost light. Even a small fraction of light from the inner corona being scattered to higher altitudes can significantly modify the observed brightness and make the interpretation of results difficult. The contribution of stray light complicates diagnostics of plasma temperature and electron density in coronagraphic (Wang et al. 2017), imaging (Shearer et al. 2012; Goryaev et al. 2014), spectroscopic (Dolla \& Solomon 2008; Hahn et al. 2011; Wendeln \& Landi 2018), and even radio (Högbom 1974; Weliachew et al. 1985) observations. Furthermore, the presence of stray light in coronal images also reduces contrast and thus precludes small-scale features from being identified.

For white-light coronagraphic observations, diffraction plays an especially important role in stray light generation. In internally occulted coronagraphs the light diffracted by the entrance aperture produces an intense, sharply decreasing stray light pattern on the detector. The intensity of the diffraction in the region of the inner corona becomes greater than the intensity of the corona itself (Rougeot et al. 2017). Furthermore, the situation is worsened by the scattering of direct sunlight on the primary objective (e.g., Thompson et al. 2010). Externally occulted coronagraphs place the primary objective (PO) in the umbra of the external occulter, which alleviates the problem of scattering, but provides no improvement of diffraction in the inner-most corona (see Fig. 10 in Rougeot et al. 2017). The remaining diffraction is removed by the internal occulter (IO), which is one of the key optical elements of externally occulted coronagraphs. The IO is the conjugated element to the EO with respect to the PO, it is slightly oversized in order to block the diffraction ring produced by the EO. Finally, on the detector the diffracted light has the shape of a bright ring with the angular size of the IO, and its full intensity is determined primarily by the IO oversizing. The intensity of diffraction in externally occulted coronagraphs is usually smaller than in internally occulted ones, however external occulters produce significant vignetting of the inner field of view (FOV; Koutchmy 1988; Bayanna et al. 2011; Rougeot et al. 2017). This explains the relatively high innermost observational heights of white-light coronagraphs. 


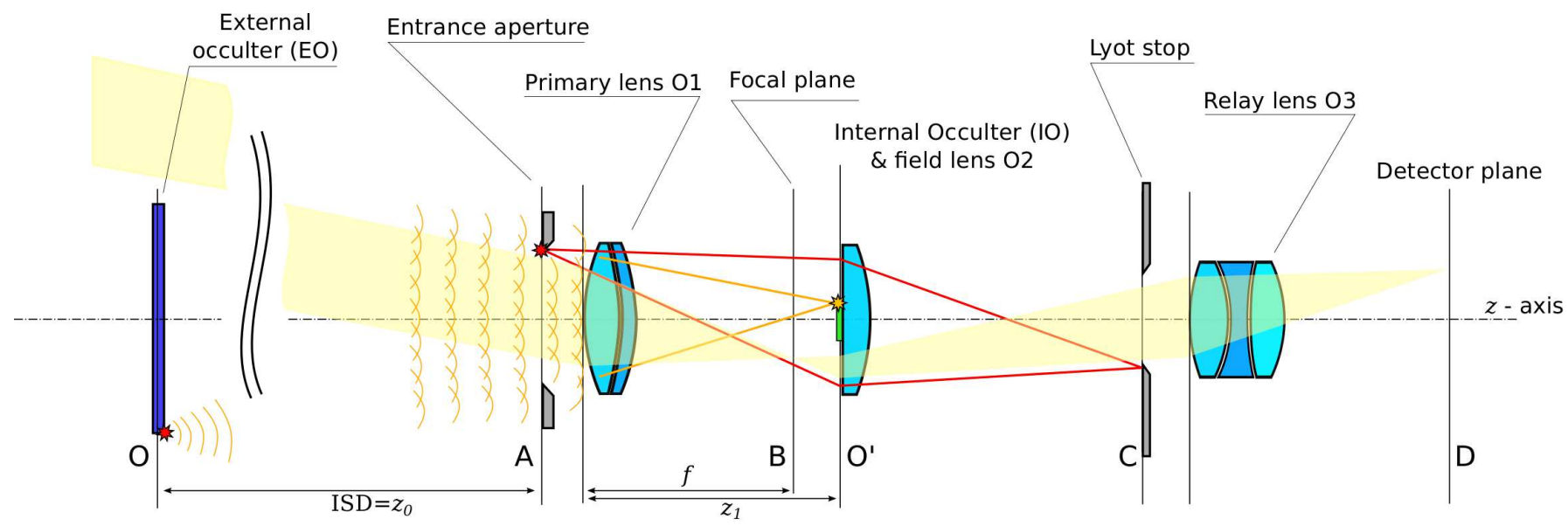

Fig. 1. Functional optical layout of the ASPIICS coronagraph. Dim yellow light represents regular coronal beam, orange arches and lines - light that diffracts on EO, the red lines represent the light diffracted at the entrance aperture.

ASPIICS (Association of Spacecraft for Polarimetric and Imaging Investigation of the Corona of the Sun) is a novel whitelight solar coronagraph that will make regular observations of corona within the FOV from $\sim 1.08 R_{\odot}$ up to $3.0 R_{\odot}$ (Lamy et al. 2010; Renotte et al. 2015; Galano et al. 2018). This will be possible owing to the European Space Agency's (ESA) formation flying (FF) mission PROBA-3 (Project for On-Board Autonomy, Bernaerts et al. 2002), in which the external occulter will be placed on the first satellite, and the telescope will be placed on the second (Leyre et al. 2005; Vivès et al. 2006).

Good performance of white-light coronagraphs requires the reduction of stray light. This usually includes the optimization of the shape of the external occulter, as in LASCO C2 (Bout et al. 2000) or METIS on board Solar Orbiter (Landini et al. 2017), the introduction of specialized diaphragms like the Lyot spot in LASCO C2 (Brueckner et al. 1995; Llebaria et al. 2004), the utilization of super-polished lenses and mirrors like in METIS (Sandri et al. 2018), and COR1 (Thompson et al. 2003), and the use of a set of baffles like in HI-1 and HI-2 onboard STEREO (Eyles et al. 2009) and so on.

In ASPIICS, various means are also used in order to reduce the level of stray light. The EO edge will have a toroidal shape, which will reduce the intensity of the diffracted light entering the telescope by a factor of two (Landini et al. 2010; Rougeot et al. 2018a); technological limitations do not allow the use of superior EOs such as those that are multi-disk, serrated, threaded cone, or even petal-shaped (see Bout et al. 2000 for a review of occulter systems, and the recent analytical/numerical analysis of serrated occulters by Rougeot \& Aime 2018). The lenses will have highquality surfaces with microroughness better than $\sim 1 \mathrm{~nm}$ and will be covered with anti-reflection (AR) coatings with $R \sim 0.3 \%$; the size of the IO will be chosen to achieve balance between the level of diffracted light and minimal observational height (Shestov \& Zhukov 2018). On-ground data processing can yield further improvement of registered data. For example, special analyses have been undertaken to model stray light in COR1 (Thompson et al. 2010), LASCO C2 (Llebaria et al. 2012), and HI-1 (Halain et al. 2011).

The expected lifetime of ASPIICS requires thorough analysis of various stray-light contributions and the algorithms for their removal. A preliminary comparison of various sources of stray light in ASPIICS showed that the most significant contribution is from ghost images. The essential part in the ghost signal is produced by the bright light of the inner corona, which initially undergoes unwanted reflection from the detector (we will use the term backreflection) and afterwards from one of the neighbouring optical surfaces.

The aim of the present paper is to analyze the behavior of optical ghost images, and compare their contribution with other sources of stray light. We develop a geometrical/mathematical model of ghost images and provide an algorithm/software for the removal of the effect.

The paper is structured as follows: in Sect. 2 we describe the optical layout of ASPIICS, in Sect. 3 we discuss possible sources of stray light, and in Sect. 4 we consider the mechanism of formation of ghost images and their characteristics. In Sect. 5, we analyze ghost images for realistic coronal scenes (both synthetic and observed during a total eclipse) and discuss a procedure for the ghost light removal. In Sect. 6 we compare the contributions of ghost images and other sources of stray light and in Sect. 7 we discuss our results and draw conclusions.

\section{Optical layout}

Below we give a description of two models: a simplified one that shows functional purposes of optical elements, and a full one, which provides further details.

\subsection{Functional optical layout}

The functional optical layout of the ASPIICS coronagraph is given in Fig. 1. The external occulter (plane $O$ ) with $₫ 1420 \mathrm{~mm}$ is situated on the occulter satellite $z_{0}=144348 \mathrm{~mm}$ ahead of the coronagraph satellite, which carries the optical instrument. The telescope consists of an entrance aperture with $\emptyset 50 \mathrm{~mm}$ (plane A) and a primary lens $\mathrm{O} 1$ (a cemented doublet) with focal length $f \approx 330.3 \mathrm{~mm}$ that makes an image of the corona in the primary focal plane $B$. The light diffracted at EO is focused in the $O^{\prime}$ plane, which is the conjugate to the plane $O$. The $O^{\prime}$ plane is situated at a distance $z_{1}$, or $\sim 0.76 \mathrm{~mm}$ further than $B$. The major part of the diffracted light is cut out by the internal occulter, the radius of which must be carefully chosen to obtain a compromise between good rejection of diffraction, minimum observational height, and vulnerability of the coronagraph to possible misalignments, such as tilt of the telescope, displacement of the IO from its nominal position, and so on (Rougeot et al. 2017; Shestov \& Zhukov 2018). The IO is deposited directly on the surface of the field lens $\mathrm{O} 2$ (a singlet lens). Lenses $\mathrm{O} 2$ and $\mathrm{O} 1$ make an image of the entrance aperture on the $C$ plane, where the Lyot stop is placed. The Lyot stop is slightly undersized ( $97 \%)$ 


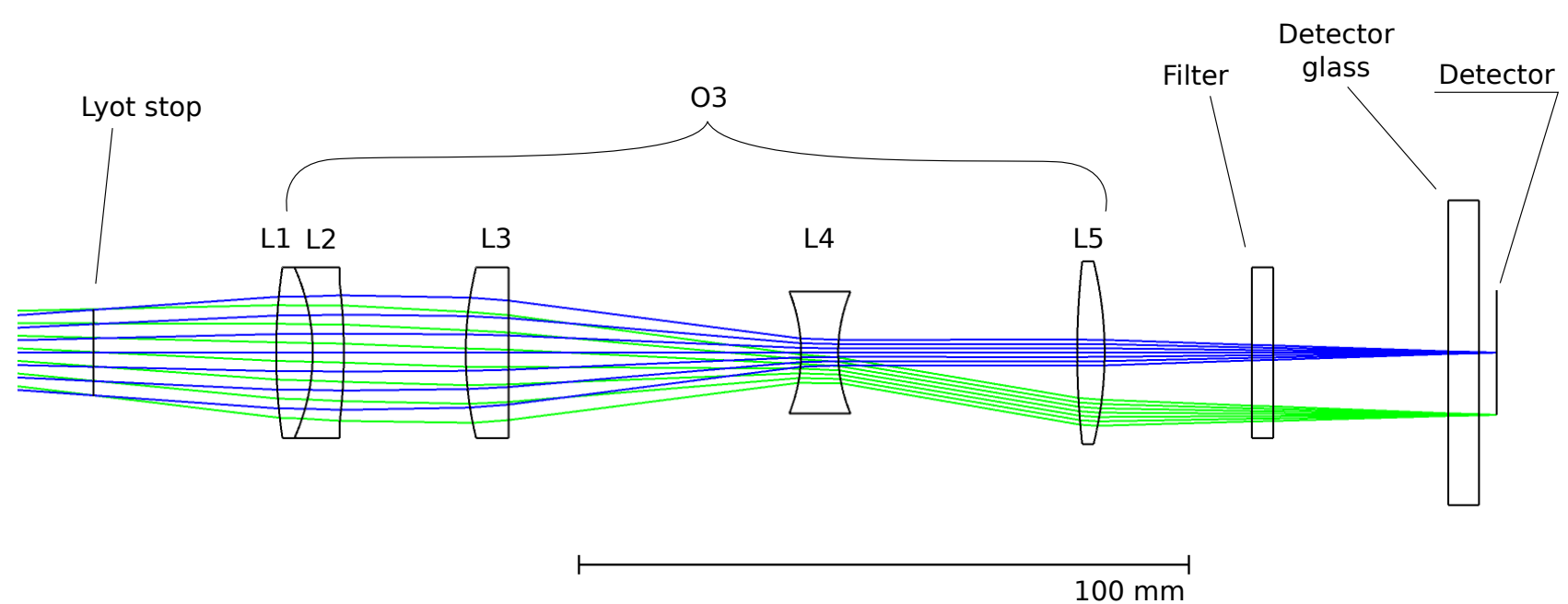

Fig. 2. Detailed optical layout of the rear part of the ASPIICS coronagraph. Blue and green lines denote ray paths propagating along the optical axis (blue) and at the edge of the FOV $-0.8^{\circ}\left(3 R_{\odot}\right)$ (green).

with respect to the entrance aperture in order to reject the light diffracted at the entrance aperture. Simultaneously, the O2 lens projects the entrance aperture to the relay lens $\mathrm{O} 3$, ensuring that the light propagates further into the coronagraph, thus justifying its name: the field lens. Finally, both $\mathrm{O} 2$ and $\mathrm{O} 3$ project the primary focus $B$ onto the detector plane $D$, making the effective focal length $f_{\text {eff }} \approx 734.6 \mathrm{~mm}$. An extensive discussion of the functional optical layout is given in Rougeot et al. (2017) and Shestov \& Zhukov (2018).

In Fig. 1, dim yellow light represents regular coronal beam, orange arches represent light that diffracts on the EO. After propagation through the entrance aperture and $\mathrm{O} 1$, this light is focused (orange lines) in the $O^{\prime}$ plane. The red lines represent the light diffracted at the entrance aperture.

\subsection{Detailed optical layout}

The real optical layout differs mainly in the more complicated relay lens $\mathrm{O} 3$, the presence of spectral filters (a filter wheel places one of the six filters into the beam), and the presence of the detector glass. A detailed description of the optical layout of ASPIICS is given in Galy et al. (2015). In Fig. 2 we provide the rear part of the optical layout of ASPIICS produced with Zemax OpticStudio.

The detector is a front-side illuminated CMOS detector with $2048 \times 2048$ pixels, each pixel being $10 \times 10 \mu \mathrm{m}$. Its geometrical size determines the outer FOV of the coronagraph up to $3 R_{\odot}=0.8^{\circ}$. The detector glass is a plane-parallel BK7 plate with a neutral-density $50 \%$ filter; it is situated $\sim 5 \mathrm{~mm}$ ahead of the detector chip and is used to reduce the relative contribution of the ghost light.

The six filters are installed in the filter wheel, which can put any one of them into the optical beam. The filters are: one wideband filter for the spectral range $535-565 \mathrm{~nm}$, two narrowband filters for $530.4 \mathrm{~nm}$ (Fe XIV) and $587.7 \mathrm{~nm}$ (He I), and three polarizers rotated by $60^{\circ}$ with respect to each other combined with the wideband spectral filters. Each filter is either a single plane-parallel glass or several glasses stacked together (like in the case with the polarizers) with AR coatings on the sides. In the current analysis we represent the filter as a single plane-parallel glass situated $\sim 40 \mathrm{~mm}$ ahead of the detector.

The relay lens $\mathrm{O} 3$ consists of five individual lenses. The last lens - O3/L5 is a telecentric lens, which re-images the entrance aperture to $z \approx-1260 \mathrm{~mm}$. This is done to improve performance of the spectral filters by reducing the range of incident angles both for an individual beam, and for the whole FOV.

\section{Sources of stray light}

A preliminary analysis of various contributors of stray light (Galy et al. 2018) demonstrated the significance of ghost light. The analysis was based on the raytracing approach and took into account such effects as ghost light and scattering on the lens surfaces. A thorough analysis of the diffracted light was performed by Rougeot et al. (2017) and Shestov \& Zhukov (2018); we summarize their findings below.

The relative importance of the ghost images is explained by the fact that the light from the innermost and thus brightest corona is re-imaged by ghost reflections to higher heliocentric heights, that is, towards the outer FOV. The intensity of the ghosts in the outer FOV regions can be as high as $10 \%$ of the intensity of the local corona. To reduce the impact of this ghost light, an additional ND50\% was introduced in the detector glass. With the presence of the ND filter the regular coronal beam loses $50 \%$ of its energy, while the ghost light is decreased by factor eight (Galy et al. 2018), passing through the filter twice more.

The light scattered on the lens surfaces provided a smaller contribution to the final stray light. The scattering has a higher efficiency at smaller angles and lower efficiency at larger angles. Therefore, scattering of the bright light from the inner corona to the greater heights was less efficient. An advanced investigation of the lens scattering characteristics is currently being performed (Rougeot et al. 2018b).

Diffracted light has the shape of a very bright and defocused ring, the size and the position of which correspond to the edge of the IO projected to the detector plane (Rougeot et al. 2017; Shestov \& Zhukov 2018). Since the effect of diffraction consists in interference of wavefront regions propagating through the opening in the apertures (Huygens-Fresnel principle), the resulting diffraction weakly depends on the lens characteristics, such as geometry and roughness, which is opposite to the behavior of ghost and scattered light. In the present analysis diffracted light is considered as an additional light source, which enters the telescope along with the coronal light, and undergoes the same ghost reflections and scattering as the coronal light does. The amount of the diffracted light incident on the primary objective is 


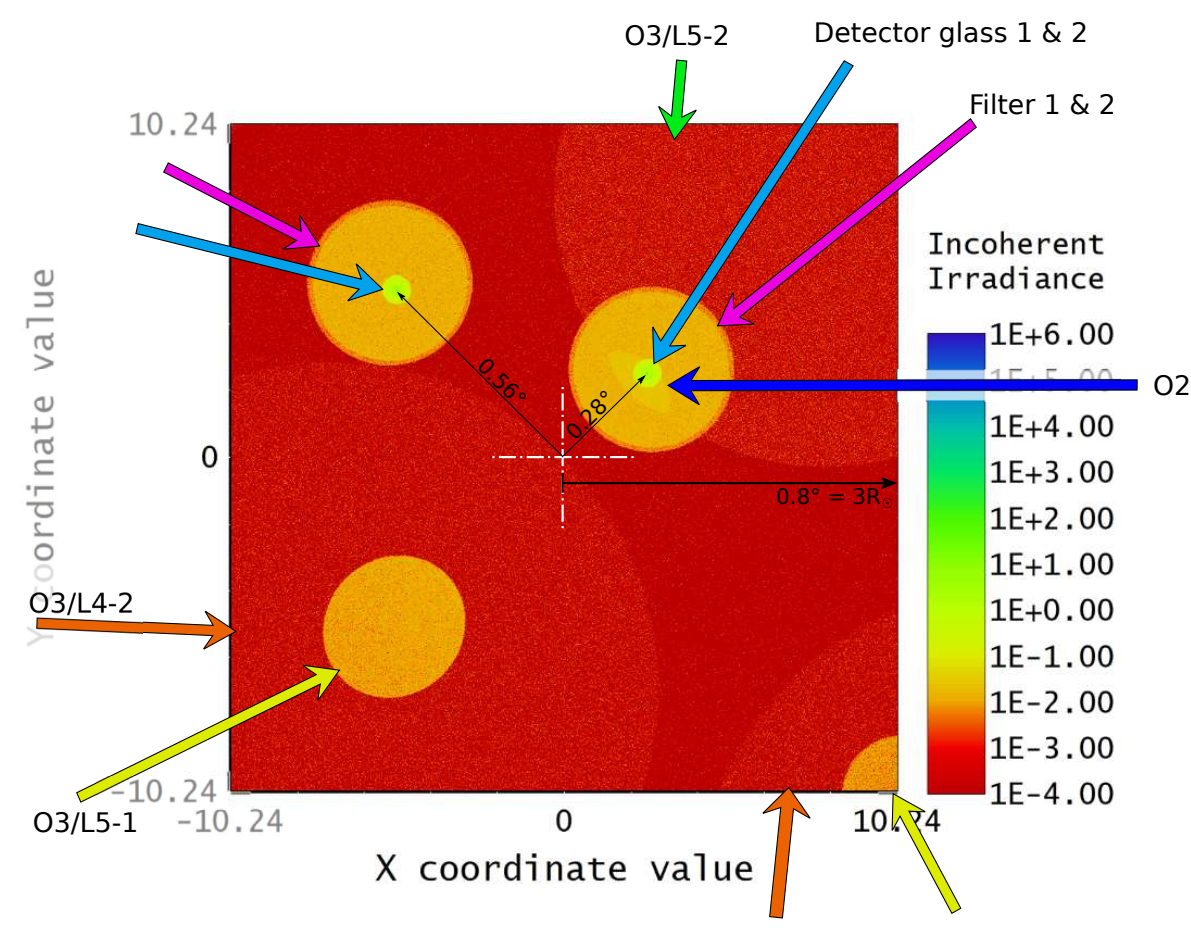

Fig. 3. Image on the detector produced in Zemax OpticStudio in nonsequential regime with two input beams tilted at $0.28^{\circ}$ and $0.56^{\circ}$. The white cross denotes the optical axis (center of the FOV), the input beams are marked with thin black arrows, and color arrows show ghost contributors produced by the backreflection from the detector and particular optical surfaces. Annotated color arrows correspond to the ghosts produced by the $0.28^{\circ}$ input beam, and the remaining arrows correspond to the ghosts produced by the $0.56^{\circ}$ beam. significantly - up to 3 orders of magnitude - larger than the amount of diffracted light coming to the detector. This is explained by the fact that a major part of diffraction is blocked by the IO. Therefore, particular attention should be paid to the analysis of ghost reflections and scattering in the primary objective.

A preliminary analysis demonstrated that the contribution of other stray light sources is negligible (Galy et al. 2018).

\section{Ghost image formation}

\subsection{Identification of the ghosts}

In order to analyze the properties of ghost images, we implemented an optical model of ASPIICS in a raytracing software package - Zemax OpticStudio in a nonsequential regime (see description of various regimes of Zemax in Appendix A). Since at this first stage of analysis we are interested in identification (determining of the parent surfaces) and general geometrical properties (size, location) of ghost images, we do not take into account any AR coatings. The reflection coefficients were calculated automatically based on glass properties and amounted to $4 \%$ for lenses, while the detector reflectivity was taken to be $15 \%$. We also do not implement the IO in the optical layout; its effect may result in additional partial/full blocking of ghost images formed by the primary objective.

We used two beams tilted at $0.28^{\circ}\left(1.05 R_{\odot}\right)$ and $0.56^{\circ}$ $\left(2.1 R_{\odot}\right)$ as an input and calculated the image on the detector with ghost reflection enabled in Zemax. We analyzed the image on the detector paying attention to the second-order ghosts, that is, the ghosts formed as a result of two reflections, which is justified by the small amount of reflected light. Since in the nonsequential regime, Zemax preserves the information about how a particular ray was formed, or which surfaces it passed through, we were able to reveal the ghost rays that were formed by individual surfaces. We were thus able to identify the origins of the ghosts, as well as their geometry and spatial behavior.
A complex image showing two input beams and ghost images created due to backreflection from the detector and another optical surface is shown in Fig. 3. The positions of the input beams are marked with thin black arrows. The reflections from both surfaces of the detector glass generate tiny circular images (blue arrows) co-centered with the input beams. The reflections from the filter surfaces yield the larger circular images (magenta arrows) almost co-centered with initial beams. The co-centering of these ghosts is due to the effect of the telecentric lens L5, which re-images the aperture almost to infinity, making the chief ray nearly perpendicular to the detector surface. The rear surface of the O3/L5 lens (O3/L5-2) deflects the beam and changes its convergence, and its ghost image (green arrow) therefore has a significantly larger diameter and is not co-centered with the input $0.28^{\circ}$ beam. For the $0.56^{\circ}$ beam, this ghost falls outside the detector. The front surface of the O3/L5 lens (O3/L5-1) also changes the convergence and deflects the beams symmetrically with respect to the optical axis. Its ghosts are shown by yellow arrows in Fig. 3. The rear surface of the O3/L4 lens produces deflected and enlarged images (orange arrows in Fig. 3), whereas the front surface of this lens produces a very large image uniformly covering the whole detector. The $\mathrm{O} 2$ lens produces a complex image that resembles the images from the detector glass and a fish-like structure (a dark blue arrow) seen near the $0.28^{\circ}$ input beam. Most of these ghosts (except for the fish-like contribution of the $\mathrm{O} 2$ ) are not vignetted inside the telescope.

In Fig. 4 we show ray paths produced by the front surface of the filter and the rear and front surfaces of the O3/L5 lens. The two bottom panels show the deflection and change of convergence by the two surfaces of the O3/L5 lens.

We paid special attention to the ghosts produced by the primary objective. The O1/L1 and O1/L2 lenses produce extremely weak and uniform illumination on the detector. Obviously, this is explained by the ghost focus position far from the detector and the beam divergence, due to which only a small portion of the rays ultimately reach the detector. The total ghost energy 
produced by the $\mathrm{O} 1$ lens on the detector amounts to $0.03 \%$ of the main ghost contributions. Such ghost behavior is in stark contrast with the LASCO C2 and LASCO C3 coronagraphs onboard SOHO (Brueckner et al. 1995; Llebaria et al. 2004), where an additional opaque disk called the Lyot spot was placed in front of the relay lens. The Lyot spot prevented the ghost created in the primary objective from further propagation. These ghosts were created in the primary objective by the light diffracted at the external occulter placed $\sim 820 \mathrm{~mm}$ (in LASCO C2) ahead of the primary objective. In the case of ASPIICS, the EO is placed $\sim 150 \mathrm{~m}$ ahead of the primary objective and the corresponding ghosts are formed between the primary objective and the field lens $\mathrm{O} 2$. The divergence of the ghost beams permits only a negligible part of the energy to propagate farther into the optical system. The possible influence of intense diffracted light falling on the primary objective with an energy 1000 times higher than the energy of the diffracted light on the detector is alleviated by the extreme loss (factor $3 \times 10^{-4}$ ) of energy of the corresponding ghost.

The total energy of the $\mathrm{O} 2$ contribution amounts only to $14 \%$ of the detector-reflected ghosts, and since the relative intensity of the fish-like component (Fig. 3) is small, the contribution of the $\mathrm{O} 2$ lens can be included in the ghost energetics of the detector glass. The full ghost energy (with any second surface, not necessarily the detector) produced by the $\mathrm{O} 1 / \mathrm{L} 1$, O1/L2, O3/L1, O3/L2 and O3/L3 lenses amounts to $0.01 \%$, $0.02 \%, 0.14 \%, 0.51 \%$, and $0.9 \%$, respectively, of the total energy produced by the detector backreflections. These lenses are therefore omitted from further consideration. The relative contribution of other ghosts, such as higher-order ghosts or second-order ghosts produced by O3/L4, O3/L5, the filter, or the detector glass (i.e., excluding the detector backreflections), is also relatively small. In the current model, with no AR coatings, the intensity of the ghosts created by the detector backreflection amounts to $61 \%$ of all ghosts, and most of the remaining $39 \%$ is due to ghost reflection by the detector glass and the filter. The introduction of a model AR coating with $R=0.5 \%$ reflectivity on several surfaces closest to the detector immediately increases this ratio to $90 \%$ or better, because the relative contribution of other ghosts decreases. Therefore, we conclude that the presence of a high-reflectivity AR with $R \sim$ $0.3 \%$ coating will make the contribution of the detector ghosts dominant.

Another effect to consider is that after reaching the detector, all ghost light will be reflected once again, and after that will produce additional ghosts following similar geometrical rules. However, the intensity of these ghosts will be small because of the small value of the reflection coefficient. In Sect. 5.2 we confirm the validity of this assumption.

We conclude that the L4 and L5 lenses of O3, the filter, and the detector glass are the primary contributors to ghost light. Their geometrical properties and energetics are further analyzed and modeled below.

\subsection{Geometrical behavior and energetics of the ghosts}

In Sect. 4.1 we used the nonsequential regime of OpticStudio to produce the ghost image for a given input image. In order to analyze the geometrical behavior of the selected ghosts depending on the incoming beam angle, we used the sequential regime of Zemax and retrieved first-order parameters of ghosts (which are obtained in a paraxial approximation; see Appendix A). Thus for each pair of optical surfaces we obtained the effective ghost focal distance $f_{\mathrm{g}}$ and its working $\mathrm{f} / \#$ ratio, the position of the ghost
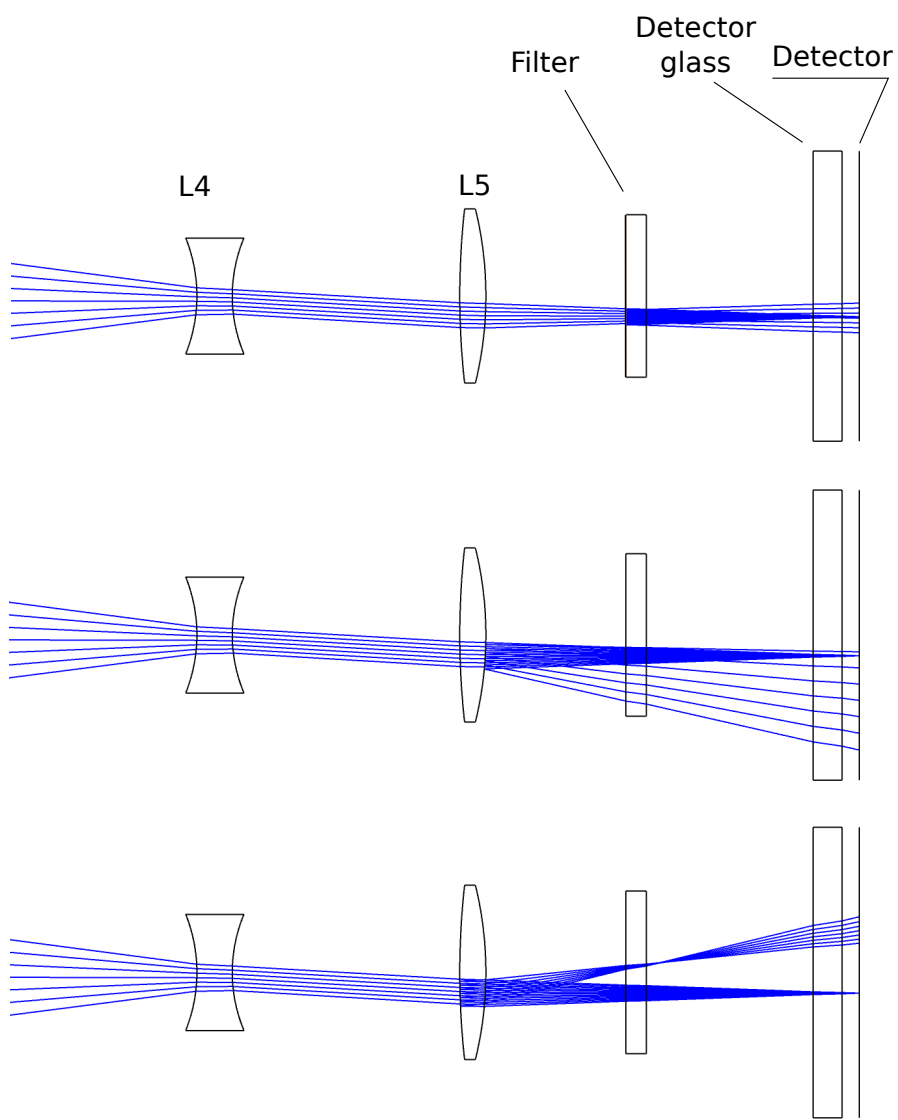

Fig. 4. Ray paths produced by an incoming beam and a ghost beam formed by the detector and one of the optical surfaces. The incoming beam is backreflected from the detector, travels back almost the same way, experiences ghost reflection and then travels back forming a different path. Top panel: ray paths produced by the filter, middle panel: those produced by the rear surface of the O3/L5 lens, and bottom panel: those produced by the front surface of the O3/L5 lens.

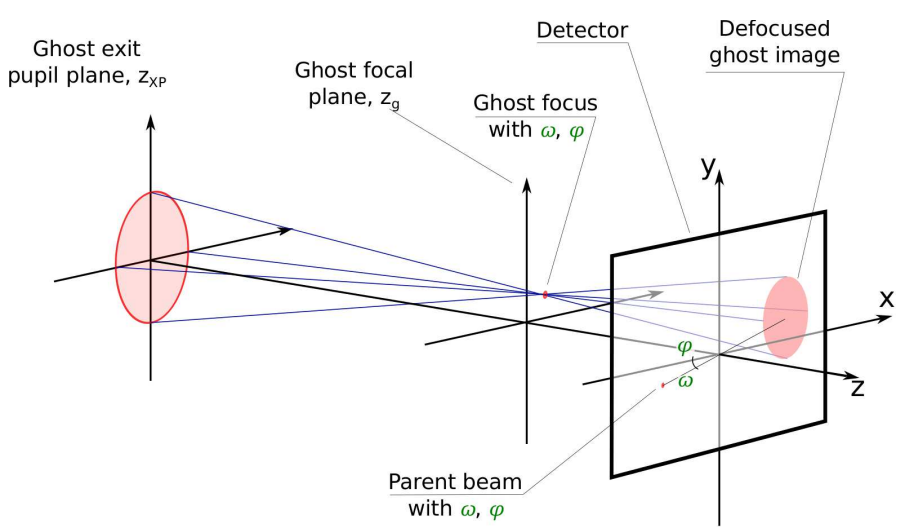

Fig. 5. Sketch representing the geometry of the defocused image of a ghost. Configuration of the parent beam and the defocused image correspond to the O3/L5-1 lens surface.

exit pupil $z_{\mathrm{XP}}$, and the position of the ghost focal plane $z_{\mathrm{g}}$. A sketch representing the formation of the defocused ghost image on the detector is shown in Fig. 5. The center of the ghost image is determined by the position of the ghost focus and the ghost exit pupil, while the diameter of the defocused image is determined by the working $f / \#$ ratio and distances between different planes. The main characteristics of the major ghosts are summarized in Table 1. 
Table 1. Characteristics of the main ghosts, formed by the backreflection from detector and another optical surface.

\begin{tabular}{llllllll}
\hline \hline Surface & Symbol & $z_{\mathrm{XP}}, \mathrm{mm}$ & $z_{\mathrm{g}}, \mathrm{mm}$ & $f_{\mathrm{g}}, \mathrm{mm}$ & $f / \#$ & $R_{\mathrm{det}}, \%$ & $R, \%$ \\
\hline Detector glass, front & $\mathrm{G} 1$ & -1273.72 & -12.19 & 731.88 & 14.64 & 15.0 & 0.3 \\
Detector glass, rear & $\mathrm{G} 2$ & -1267.14 & -5.60 & 731.88 & 14.64 & 15.0 & 0.3 \\
Filter glass, front & $\mathrm{F} 1$ & -1335.83 & -74.30 & 731.88 & 14.64 & 15.0 & 0.23 \\
Filter glass, rear & $\mathrm{F} 2$ & -1331.22 & -69.69 & 731.88 & 14.64 & 15.0 & 0.23 \\
Relay objective, L5 lens front & O3/L5-1 & -41.71 & -30.46 & $-385.98^{a}$ & 7.72 & 15.0 & 0.23 \\
Relay objective, L5 lens rear & O3/L5-2 & -92.22 & -82.23 & 249.06 & 4.98 & 15.0 & 0.23 \\
Relay objective, L4 lens rear & O3/L4-2 & -132.01 & -114.02 & $-282.36^{a}$ & 5.65 & 15.0 & 0.23 \\
\hline
\end{tabular}

Notes. $z_{\mathrm{XP}}$ - exit pupil position, $z_{\mathrm{g}}$ - effective focus position, $f_{\mathrm{g}}$ - effective focal length, $f / \#-$ working $f / \#, R_{\mathrm{det}}-$ reflectance of the detector, $R-$ reflectance of the second surface. $z=0$ position corresponds to the detector plane, $z$-negative direction is towards the entrance aperture. Positive focal length corresponds to the situation where a ghost image is formed on the same side along with parent beam with respect to the center of the optical axis. ${ }^{(a)}$ Negative value means symmetrically with respect to the optical axis.

The total energy stored in each ghost image is determined by the intensity of the parent beam $I$, the reflectivity of the detector $(\sim 15 \%)$, the reflectivity of the particular optical surface (determined by the deposited AR coating with $R \sim 0.3 \%$ ), and the transmission of the glass elements (amounts to $\sim 70 \%$ for all the lenses). The total energy of the ghost image, however, is uniformly spread across its full area. The specific intensity (per pixel) di of a particular ghost image depends on the parent surface: the specific intensity of the detector glass ghost will be considerably higher than the intensity of the O3/L5 lens ghost due to its considerably smaller area. We have verified that all ghosts considered here (beside the fish-like contributor of the O2) do not experience additional vignetting inside the telescope.

\subsection{Geometrical model and software for calculation}

The computation of a ghost image using a raytracing approach is relatively computationally expensive: even for a simple input image with just two incoming beams, each containing $\sim 10^{5}$ rays, it takes up to $10 \mathrm{~min}$ to compute the final image using OpticStudio on a contemporary Intel i5 computer. Additionally, there are various difficulties in embedding raytracing software into an automated data-processing workflow. Therefore, we developed software for calculating the ghost image for a given input image in order to embed it into the on-ground image-processing pipeline.

We assume that every pixel of the input image represents an incoming plane-parallel wave and calculate the ghost response for every plane-parallel component. We loop through all the pixels of the input image using two nested loops in $y$ and $x$, and for every pixel we calculate its radial $(\omega)$ and polar $(\varphi)$ coordinates. Based on these coordinates we calculate the position of the ghost focus $x_{\mathrm{g}}$ and $y_{\mathrm{g}}$ in the ghost focal plane with coordinate $z_{\mathrm{g}}$ using the geometrical parameters from Table 1 . The ghost focus and the ghost exit pupil XP determine the position and the size of the defocused ghost image on the detector (see Fig. 5). In two next-level loops we check whether every pixel on the detector with the coordinates $[\mathrm{K}, \mathrm{P}]$ falls within the defocused ghost image. We perform this by verifying if the line through the points $\left(x_{\mathrm{g}}, y_{\mathrm{g}}, z_{\mathrm{g}}\right)$ and $(K, P, 0)$ crosses the exit pupil. In cases where the pixel belongs to the defocused ghost image we increase the intensity in the ghost image $G[K, P]$ by the specific intensity di (which itself depends on the intensity of the initial pixel, reflectivities, and the area of the ghost). The procedure is carried out for every pair of ghost-producing surfaces, that is, for every row in Table 1, with the following algorithm:

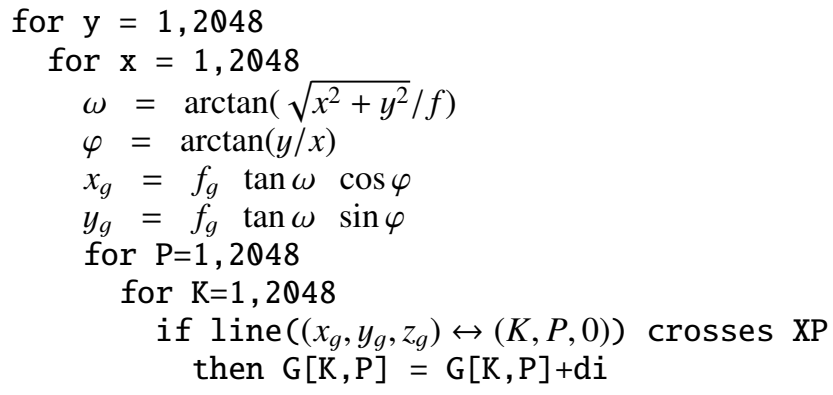

We implemented this algorithm in Fortran and fed it with all the geometrical characteristics and reflectivities. In Fig. 6 we show examples of ghost images calculated with the model for an input image with two bright pixels that represent two plane-parallel beams tilted at angles of $0.28^{\circ}$ and $0.56^{\circ}$.

Comparison of the model presented in Fig. $6 \mathrm{~b}$ with the ghost image calculated in the Zemax nonsequential regime (Fig. 3) reveals a perfect correspondence for most of the ghost contributions. We found a small mismatch of the O3/L5-1 contributions, which were slightly shifted outwards from the center and had a small ellipticity in the case of the Zemax nonsequential model. We believe the mismatch is due to the fact that geometrical characteristics of the ghosts were obtained in Zemax in a paraxial approximation. A future update of the model will take this discrepancy into account.

The procedure for calculating the ghost images from a particular surface very much resembles convolution. However, since most of the ghosts are not co-centered with the original pixel, the procedure cannot be immediately implemented as a convolution. This essentially precludes implementing the algorithm in an interpreted programming language (such as IDL, Python or MATLAB), since two nested loops would run for a very long time for a $2048 \times 2048$ image. Instead, we use a compiling programming language (Fortran) along with OpenMP parallelization. The full procedure takes up to $10 \mathrm{~min}$ for an input $2048 \times 2048$ image on a computer with 40 Xeon e5-2580 cores.

\section{Ghost image for a realistic coronal image and the ghost removal procedure}

\subsection{Ghost image for a synthetic coronal image}

The mechanism of ghost light formation and the images presented in Fig. 6 do not tell us how the effect will degrade real 


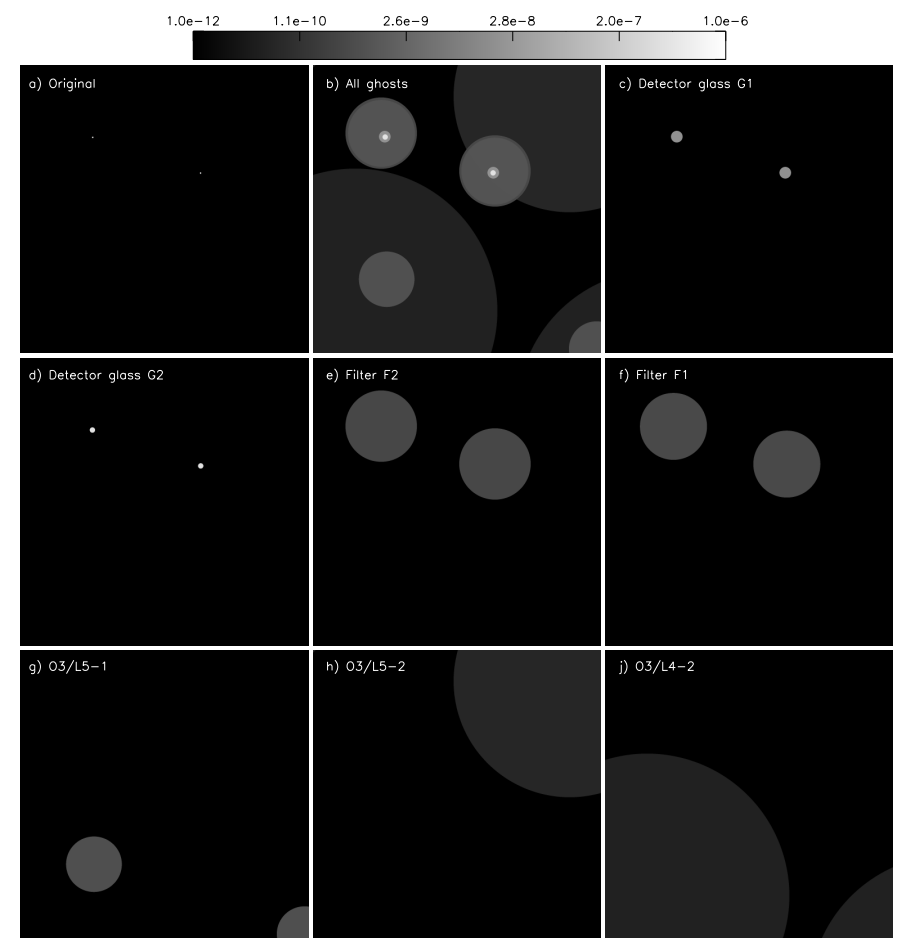

Fig. 6. Ghost images produced for an input image with two bright pixels (up-right and up-left from the center) representing two plane-parallel beams tilted at $0.28^{\circ}$ and $0.56^{\circ}$. Panels $a$ and $b$ : input image and full ghost image, respectively. Remaining panels: ghost image produced by the front surface of the detector glass (panel $c$ ), the rear surface of the detector glass (panel $d$ ), the rear filter surface (panel $e$ ), the front filter surface (panel f), the rear surface of the O3/L5 lens (panel $g$ ), the front surface of the O3/L5 lens (panel $h$ ), and the rear surface of the O3/L4 lens (panel i). Logarithmic color scale (shown at the top of the figure) is normalized to the intensities of the input beams.

observational data. In order to understand this, we calculated the ghost image for a synthetic coronal image. Such an image is presented in Fig. 7a. The synthetic image consists of two components: a synthetic coronal scene and a diffracted light scene. Inclusion of diffracted light in the input image is important because the diffraction has significant intensity in the inner part of the FOV, and as a result this light could have a significant impact on the outer part.

We created the coronal scene in the following way: initially we created a mask with an appropriate spatial scale and FOV and populated it with various structures: equatorial streamers, equatorial and polar quiet Sun regions, and finally the region that corresponds to the occulter. After that we calculated the intensity in each pixel based on the height above the solar limb and the type of coronal structure to which it belongs. The radial dependencies of intensities are taken from Allen (1976), and have typical values from $10^{-5} \mathrm{MSB}$ at heights of $\sim 1.01 R_{\odot}$ (equatorial streamers) to $10^{-10} \mathrm{MSB}$ at heights of $\sim 3 R_{\odot}$ (polar region during solar minimum). The coronal scene is vignetted in the inner zone due to vignetting produced by the IO. The diffracted light scene was calculated following the algorithm of Shestov \& Zhukov (2018) for the symmetrical ASPIICS configuration and the IO size $r_{\mathrm{IO}}=1.662 \mathrm{~mm}$. The diffraction pattern has a bright ring coinciding with the size of the $\mathrm{IO}$, and a smaller bright ring that corresponds to the internal opening in the IO.

The resulting synthetic image does not represent real corona in terms of variety of observed structures. However, it has the correct dynamic range and reasonable radial decrease of inten- sity. High contrast between structures makes it convenient for an investigation of the optical properties of the telescope.

Figure 7 compares the synthetic coronal image (panel a) and calculated ghosts (same notation as in Fig. 6). This comparison confirms the preliminary result that ghosts redistribute the light from the inner corona to the outer FOV. In particular, the effects produced by O3/L5 and O3/L4-2 introduce a significant contribution to the outer corona. The ghost image produced by the detector glass looks like a smeared version of the original; therefore, it may effectively broaden the point spread function of the telescope. It is interesting to note that the ghost images formed by the filter and lenses lose any internal structure, regardless of the fact that the input image had structures with very high contrast. This fact may open the possibility of calculating ghost images with a simplified approach based on convolutions, which may speed-up the on-ground routine processing. Another possibility could be to use a particular coronal image and calculate ghost image for a set of subsequent observations, in which the corona does not change significantly.

We compare radial profiles of the intensities of the input image and various ghost contributions in Fig. 8. In the upper panel the profiles are measured along the horizontal line (westward from the disk center in Fig. 7a) that crosses a streamer and a quiet equatorial region. In the lower panel the profiles are measured along the vertical line (northward from the disk center in Fig. 7a) that crosses the quiet polar region. The profiles in the upper panel represent the worst case, because the weak outer corona is superposed with the light from bright inner coronal structures. The main ghost contributors for the outer corona are the O3/L5 and O3/L4 lenses. In the inner corona the main contribution is from $\mathrm{G} 2$ - the rear surface of the detector glass. At the heights above $\sim 2 R_{\odot}$ the ghost intensity amounts to $3 \%$ of the coronal signal. This effect is achieved by the introduction of the ND50\% filter, which reduces intensity of ghosts by a factor four with respect to the outer corona.

\subsection{Removal of the ghost light}

The algorithm described in Sect. 4.3 provides the ghost image for a highly idealized input image, one that does not contain ghost light so far. In reality, the detector of the ASPIICS telescope will record images that contain both the image itself and the ghost light in a single observation. The problem of determining the ideal input image given the degraded image is not straightforward. However, since the intensity of the ghost image is significantly smaller than that of the input image, we can use the following approach. With I being an ideal input image, $\mathbf{G}()$ a function to calculate the ghost image, and $\mathbf{R}$ the recorded image: $\mathbf{R}=\mathbf{I}+\mathbf{G}(\mathbf{I})$. We apply the ghost function $\mathbf{G}()$ to both parts of the equation and obtain:

$\mathbf{G}(\mathbf{R})=\mathbf{G}(\mathbf{I}+\mathbf{G}(\mathbf{I}))=\mathbf{G}(\mathbf{I})+\mathbf{G}(\mathbf{G}(\mathbf{I}))$,

as $\mathbf{G}()$ is obviously a linear function. Since in every point $\mathbf{G}(\mathbf{I})_{x y}<\mathbf{I}_{x y}$, we obtain that $\mathbf{G}(\mathbf{G}(\mathbf{I}))_{x y}<\mathbf{G}(\mathbf{I})_{x y}$, and thus we can assume that:

$\mathbf{G}(\mathbf{R}) \approx \mathbf{G}(\mathbf{I})$

The validity of the approximation can be verified numerically. We calculated both the ghost image $\mathbf{G}(\mathbf{I})$ and recorded image $\mathbf{R}$ (Fig. 9a) for the synthetic input image from Fig. 7a. Based on the degraded image we calculated ghosts as $\mathbf{G}(\mathbf{R})$ (Fig. 9b) and a cleared image $\mathbf{C}=\mathbf{R}-\mathbf{G}(\mathbf{R})$. We show the difference between $\mathbf{I}$ and $\mathbf{C}$ in Fig. 9c. The difference, that is, the remaining ghost 

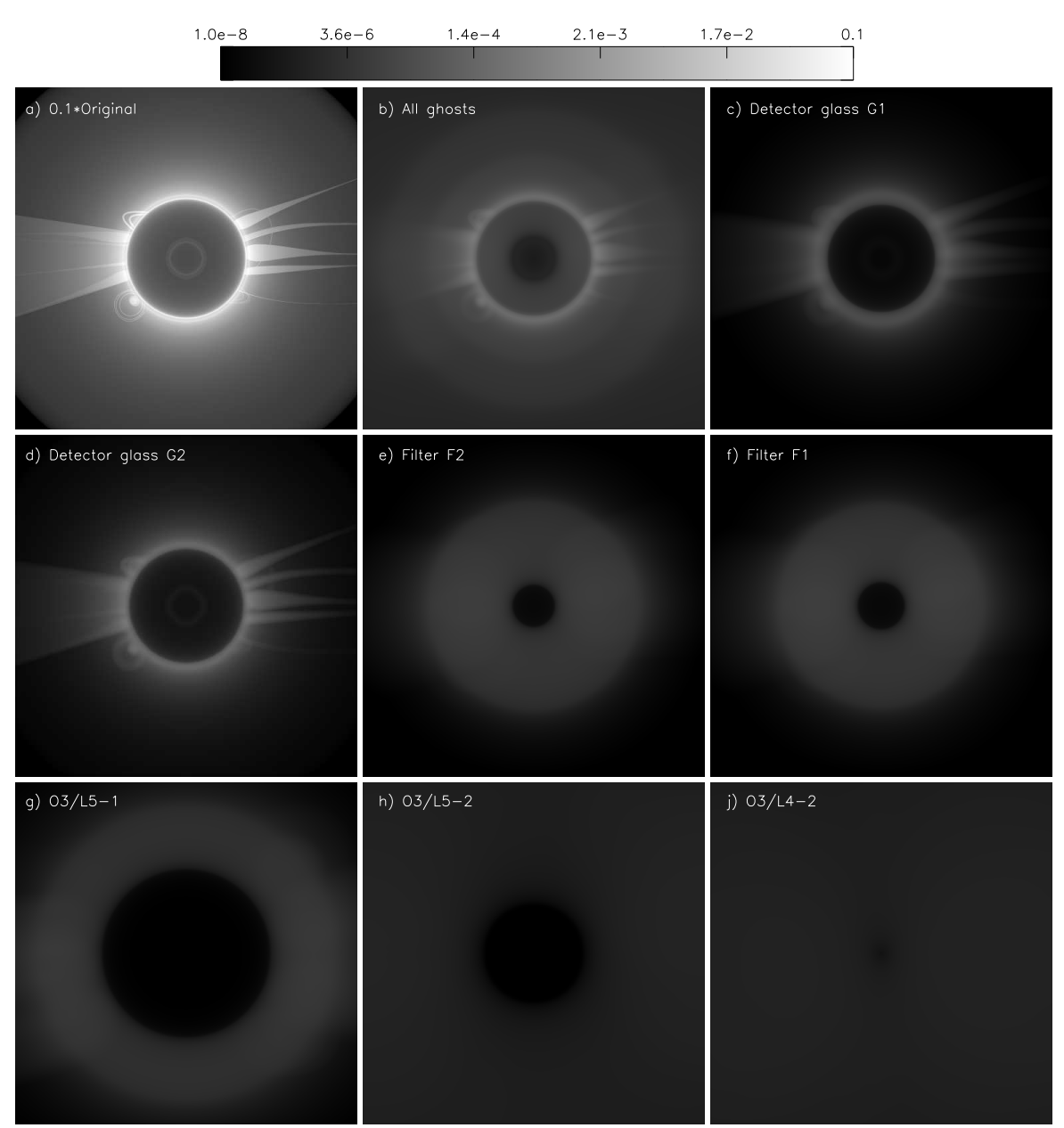

Fig. 7. Ghost images for the synthetic coronal image. Panel $a$ : input image with coronal and diffracted light superposed, other panels show different ghosts and have the same meaning as in Fig. 6. The color scale is normalized to the intensity of the input image. The intensity in panel $a$ is divided by 10 for visualization purposes. signal left after the removal procedure, is significantly weaker than the original ghost image $\mathbf{G}(\mathbf{R})$ and, on average, amounts to $10^{-5}$ of the input. Following the algorithm presented in Sect. 4.3 we calculated the ghosts of the ghosts $\mathbf{G}(\mathbf{G}(\mathbf{I}))$. The intensity turned out to be $\sim 2-3$ orders of magnitude less than the original ghost image $\mathbf{G}(\mathbf{I})$, and its spatial pattern resembled the pattern of the original pattern of the ghost. Therefore, the key assumption $\mathbf{G}(\mathbf{G}(\mathbf{I}))_{x y}<\mathbf{G}(\mathbf{I})_{x y}$ is valid with a good accuracy.

This result justifies Eq. (2) and the validity of the proposed algorithm. It also confirms that the effect of high-order ghosts is negligible (see Sect. 4.1).

\subsection{Ghost image for a real coronal image}

In this section we test the algorithm described above using a real coronal image recorded during a total solar eclipse.

These eclipse observations were obtained during the 1999 August 11 eclipse in Râmnicu Vâlcea, Romania, in an experiment originally designed to produce a high-quality, fully calibrated image that could be used to constrain stray light in images from the LASCO C1 coronagraph (Brueckner et al. 1995). In spite of the loss of $\mathrm{C} 1$ after the SOHO failure in 1998, which rendered that goal moot during the eclipse, the observation proceeded and yielded a high-quality image that captures the $530.3 \mathrm{~nm}$ Fe XV so-called coronal green line across its complete dynamic range (Seaton 2001).

These observations used a purpose-built baffled simple lens telescope (to minimize the risk of internal reflection) with a $5 \mathrm{~cm}$ diameter, $300 \mathrm{~mm}$ focal length, and focal ratio of $\mathrm{f} / 6$. Spectral selection was achieved using a Dayster $530.3 \mathrm{~nm}$-centered filter with a $0.36 \mathrm{~nm}$ passband and a peak transmission of $50 \%$. The camera was a 14-bit Photometrics PM512 CCD with a $20 \mu \mathrm{m}$ pixels, a $512 \times 512 \mathrm{FOV}$, with a measured platescale of 14.03 arcsec pixel $^{-1}$. The CCD gain was $16 \mathrm{e}^{-} / \mathrm{DN}$. To cover the entire dynamic range of the corona, which ranges more than three orders of magnitude between 1 and $4 R_{\odot}$, with adequate $\mathrm{S} / \mathrm{N}$ and no saturation, we used multiple exposure times ranging from 0.25 to $32 \mathrm{~s}$. However, we discarded the $32 \mathrm{~s}$ exposure before constructing a final composite because blooming from extreme saturation in the inner corona obscured a large fraction of the FOV.

Individual images were calibrated using flats and darks obtained immediately before and after the eclipse, and were exposure-normalized. The final image is a composite of nine separate exposures, each masked to exclude regions of saturation and regions with poor $\mathrm{S} / \mathrm{N}$. Because for this analysis we seek a smooth image with minimal noise, it is important to suppress temporal noise as much as possible. To help achieve this, each of the individual input images is treated using a local filter that replaces any pixel that exceeds the range of its eight neighbors with the local maximum (or minimum, where appropriate). The individual masked images are then combined into a single high-dynamic-range (HDR) composite by computing the median value of the inputs for each pixel.

The absolute radiometric calibration of the HDR composite result is achieved by using a separate set of reference images of the uneclipsed Sun. From these we compute the radiance per pixel of the full Sun. 

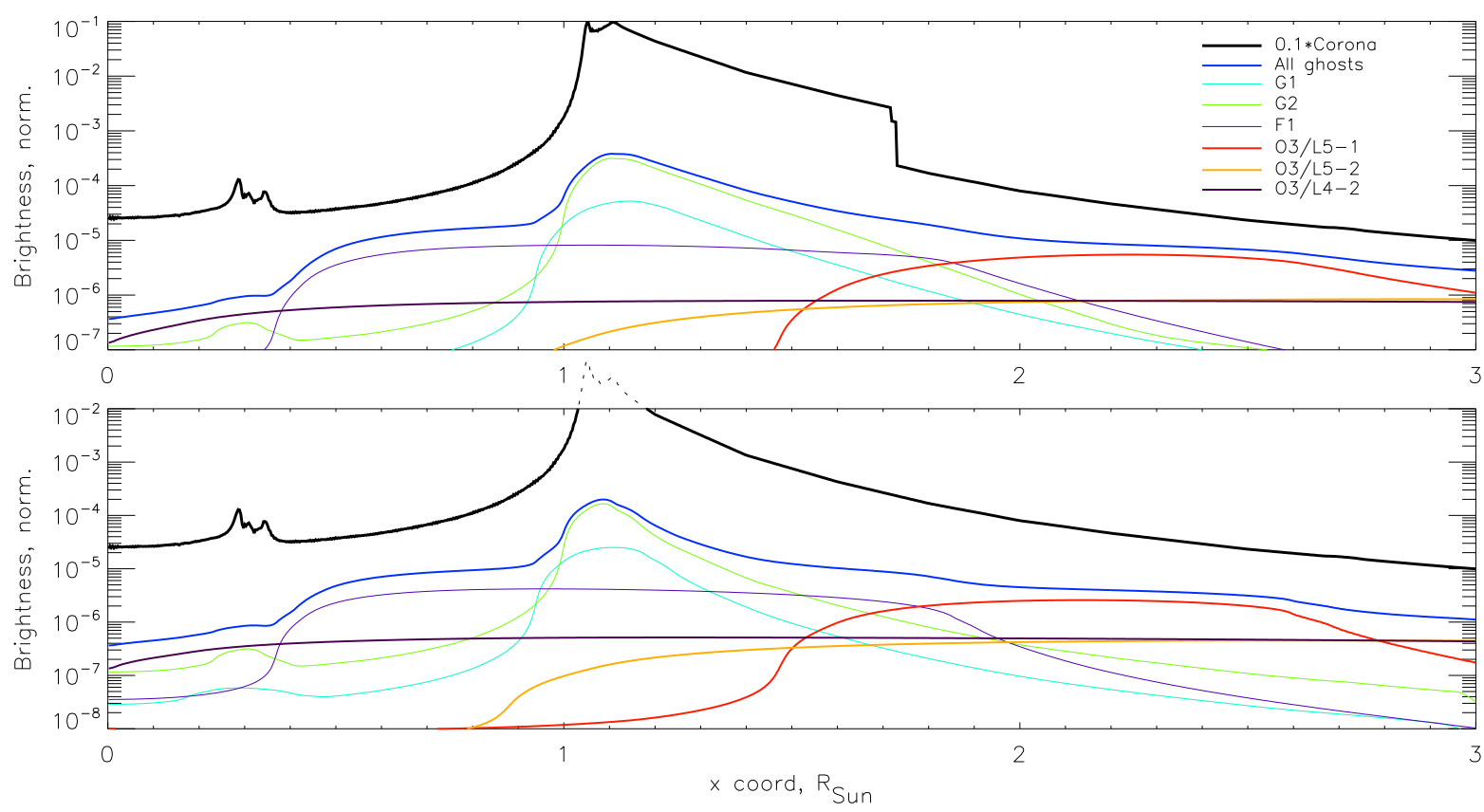

Fig. 8. Radial profiles of the intensity of the synthetic coronal image and various ghost contributions. Upper panel: westward equatorial direction in Fig. 7a that crosses the equatorial streamer and the quiet Sun region, lower panel: northward equatorial direction in Fig. 7a that crosses a polar quiet-Sun region. In both panels black solid lines show the corona and diffraction (intensity is divided by 10), dark blue lines show the sum of all ghost contribution, and other colored lines show contributions from various optical surfaces.

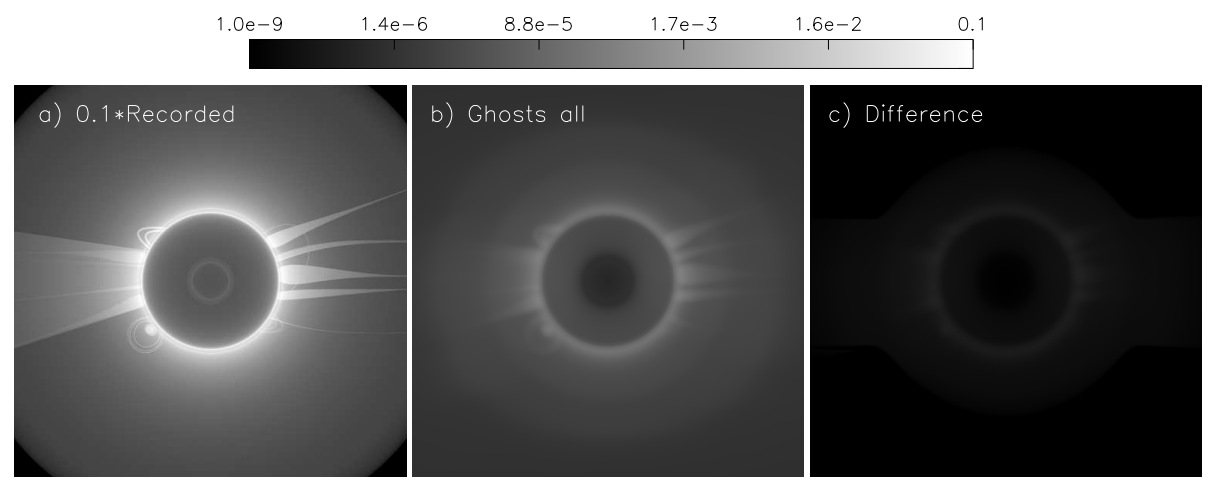

Fig. 9. Analysis of the removal procedure for the synthetic coronal image. Left panel: recorded image $\mathbf{R}=\mathbf{I}+\mathbf{G}(\mathbf{I})$. Middle panel: ghost image $\mathbf{G}(\mathbf{R})$. Right panel: difference $\mathbf{I}-$ $\mathbf{C}$ between the initial image and the cleared image. The color scale is normalized to the intensity of the recorded image. The intensity in panel $a$ is divided by 10 for the visualisation purposes.

The rate of fall-off of the green-line corona in the resulting image is roughly consistent with observations of the green line at the 1981 total eclipse by Kim (1997), which show a decrease of roughly three orders of magnitude between heights of 1 and $2 R_{\odot}$. It is worth noting that a small amount of residual signal is present in the darkest areas of the image, likely the result of scattered light inside the telescope; this is however significantly less than 1 part in 1000 relative to the radiances recorded in the inner corona, and therefore is only significant at large heights. Likewise, areas of blooming in the longest exposures rendered some faint regions nonetheless unusable for compositing, which led to a small increase in noise in some areas of the image. Again, the noise is insignificant relative to the overall observed brightness in much of the corona.

This final calibrated image is then resampled to the correct ASPIICS plate scale and resolution and the data are rescaled according to the effective area of the ASPIICS instrument.

As before, we add the diffracted light calculated for the IO with $r_{\mathrm{IO}}=1.662 \mathrm{~mm}$ and corresponding vignetting of the corona. In Fig. 10 we compare the registered image, the ghost image, and the difference with the original.
As in the case with the synthetic image, the removal algorithm performs well and the residual, uncorrected signal amounts to $10^{-5}$ of the original corona.

\section{Comparison with other sources of stray light}

In this section we compare the brightnesses of the corona with that of the various stray light contributors: diffracted light, ghost light, and scattered light. We take intensity of the corona and the diffracted light on the detector as in Sect. $5.1\left(r_{\mathrm{IO}}=1.662 \mathrm{~mm}\right.$, symmetrical ASPIICS configuration, corona is vignetted by the IO). We calculate the ghost and scattering images individually for the coronal and for the diffracted light scenes.

The calculation of the scattered light image is based on the approach and parameters presented in Galy et al. (2018). However, here we calculate scattering for a realistic coronal scene. To achieve this, we consider the effect on the detector from scattering at a particular lens as a convolution with some kernel. We obtain the kernel from the raytracing modeling and then convolve the input image with the kernel. Since the scattering on lens surfaces is rather small, with typical values of total integrated scatter (TIS; Harvey et al. 2012) being $10^{-4}$ of the 

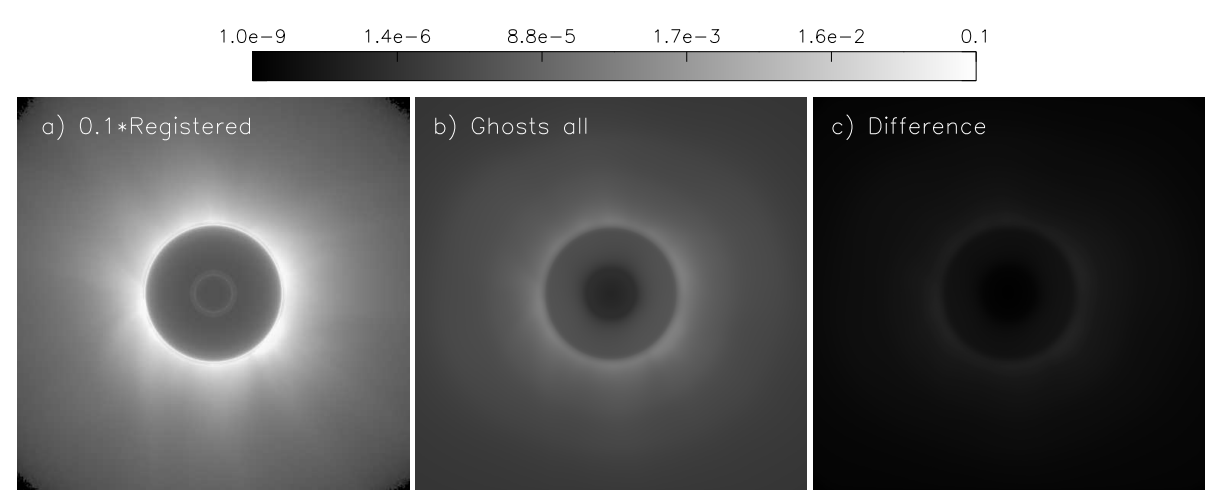

Fig. 10. Analysis of the removal procedure for the coronal image obtained during a total solar eclipse. Panels have the same meaning as in Fig. 9.

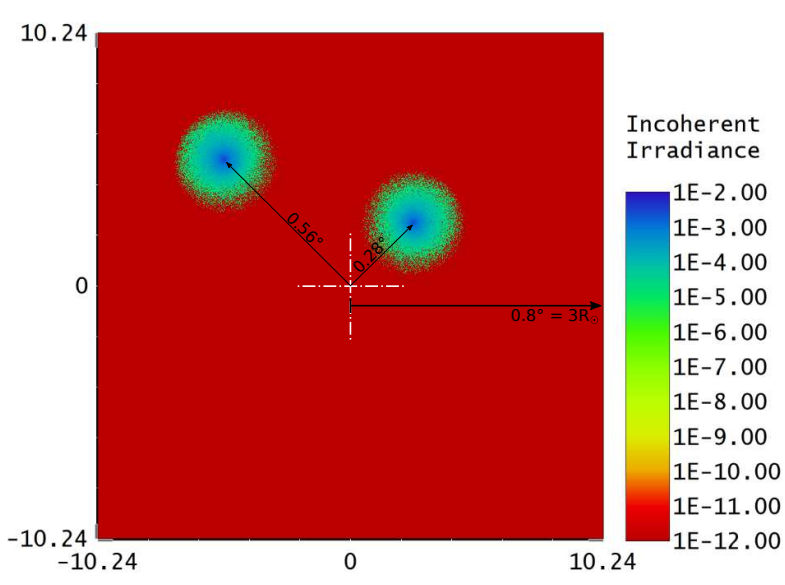

Fig. 11. Image on the detector produced in Zemax OpticStudio in nonsequential regime with scattering on the $\mathrm{O} 2$ lens for the two input beams tilted at $0.28^{\circ}$ and $0.56^{\circ}$. The logarithmic color scale corresponds to irradiance and is normalized to the peak irradiance of the scattered images.

incoming radiation, we consider scattering on each lens independently. Such an approach corresponds to consideration of the first-order scatter, in which every individual ray is allowed to scatter no more than once.

Lens scattering characteristics were inferred from the atomic force microscopy (AFM) measurements. Surface root-meansquare (RMS) micro-roughness of the lens surfaces ranged from $0.6 \mathrm{~nm}$ (both lenses of the primary objective) to $3.0 \mathrm{~nm}(\mathrm{O} 3 / \mathrm{L} 2-$ second lens of the relay lens). Based on these parameters and using an $\mathrm{ABg}$ representation (Pfisterer 2011) of the bi-direction scattering distribution function (BSDF) in the nonsequential regime of Zemax OpticStudio, we calculated a response on the detector produced for two incoming plane-parallel beams. The strongest scattered signal was produced by the $\mathrm{O} 2$ lens (see Fig. 11) and O3/L1 lens. For these lenses the following parameters were applied: RMS micro-roughness $\sigma \sim 2.6 \mathrm{~nm}$, TIS $=3 \times 10^{-4}$, and fitted $\mathrm{ABg}$ parameters $A=3 \times 10^{-5}$, $B=10^{-3}, g=1.5$. Scattering produced by the remaining optics had even smaller intensity and produced diffuse uniform images.

The image presented in Fig. 11 shows scattering produced by the $\mathrm{O} 2$ lens for the two input plane-parallel beams tilted at angles of $0.28^{\circ}$ and $0.56^{\circ}$. We fit each kernel with the analytical formula $I(r)=I_{0} \times 10^{-r / r_{0}}$. The parameter $r_{0}$ was determined to be $r_{0}=0.5 \mathrm{~mm}$, and the amplitude $I_{0}$ was adjusted to produce the total intensity of $1.3 \times 10^{-5}$ of the initial beam (corresponding to the Zemax result).

The O3/L1 lens produced a wide scattering pattern that we approximated as $I(r)=I_{0} \cdot \exp \left(-\frac{r^{2}}{2 w^{2}}\right)$. The parameters were fitted as $w=7.0 \mathrm{~mm}$, and $I_{0}$ was adjusted to produce the total intensity of $1.05 \times 10^{-5}$ of the initial beam.

We used the two kernels obtained above to produce scattering for the realistic input image containing coronal and diffraction scenes (from Fig. 7a).

Since the amount of diffracted light coming to the PO is significantly larger than that on the detector, we analyzed scattering on the PO individually. We determined scattering properties for the PO, for which we used the following parameters: RMS micro-roughness $\sim 1 \mathrm{~nm}$, TIS $=5.5 \times 10^{-5}, \mathrm{ABg}$ parameters: $A=5.5 \times 10^{-6}, B=10^{-3}, g=1.5$. Raytracing showed that the scattering was mainly determined by the O1/L1 lens, and the pattern on the detector was fitted as $I(r)=I_{0} \cdot \exp \left(-\frac{r^{2}}{2 w^{2}}\right)$, where $w=11.0 \mathrm{~mm}$, and the amplitude $I_{0}$ was adjusted to produce the total intensity $9.08 \times 10^{-7}$ of the initial beam. This kernel was convolved with the radial profile of the diffracted light corresponding to the one presented in Fig. 6 in Rougeot et al. (2017).

The results are presented in Fig. 12 showing all the contributors of stray light. The coronal profile (thick red line) corresponds to the direction westward from the disk center in Fig. 7a and crosses a streamer and a quiet region. The ghost light was calculated for the corona and the diffracted light (which includes only the intensity on the detector, since the intensity of ghosts produced by $\mathrm{O} 1$ is negligible). The scattered light contains components produced by $\mathrm{O} 1$ and strong incoming diffracted light, as well as contributions from $\mathrm{O} 2$ and $\mathrm{O} 3$ produced by both coronal light and the diffracted light signal.

Figure 12 demonstrates that the scattering has a smaller contribution than the ghost reflection and diffraction. Further, the contribution of ghosts is smaller than that of the diffracted light; we note, however, that the diffracted light given here is calculated for an "average" case - for the symmetrical configuration (tilt of the telescope would increase the intensity of the diffraction ring in one side; see Shestov \& Zhukov 2018) and a rather small $r_{\mathrm{IO}}=1.662 \mathrm{~mm}$. Comparison of the green and red curves demonstrates that the ghost contribution due to diffracted light will be smaller than the corona. The major contribution of the scattered light is produced by $\mathrm{O} 1$. This contribution is rather uniform across the detector, whereas the smaller contribution from $\mathrm{O} 2$ and $\mathrm{O} 3$ lenses has a relatively more intense central part.

\section{Discussion and conclusions}

Here, we analyzed sources of the ghost light on the ASPIICS detector. We showed that due to the relatively high reflectivity of the detector $(15 \%)$ and low reflectivity of the AR coatings $(\sim 0.3 \%)$ the main ghosts are produced by the backreflection from the detector and the neighboring optical surfaces. These contributions are relatively easy to model because of the 


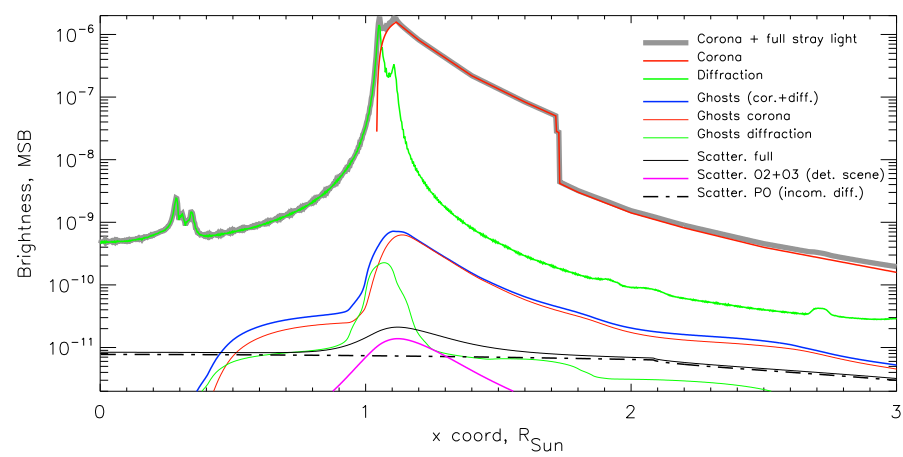

Fig. 12. Radial profiles of the intensity of the coronal light, the diffraction, ghost, and scattered light contributions. The thick red line corresponds to the corona, the thick green line corresponds to the diffraction, and the thick gray line corresponds to the full signal on the detector. The dark blue line represents ghosts of the full image, and the thin red and green lines are contributions due to coronal ghosts and diffracted light ghosts. The thin black line shows full scattering light, and the dot-dashed line and magenta line show contribution from the primary objective (dot-dashed) and the $\mathrm{O} 2$ and $\mathrm{O} 3$ lenses (magenta line).

simple geometrical behavior and absence of vignetting of the ghost beams inside the telescope. Redistribution of the light from the inner corona to the outer corona caused by the ghost reflections makes the effect important for observation of the outer corona. Currently, with the presence of the ND50\% filter, the ghost signal amounts to $3 \%$ of the coronal signal.

We created a model that calculates the ghost image for a given input image. The major ghost contributors that are not taken into account in the current model are created by the filter and the detector glass and the other optical surfaces. The ghost image calculated for a synthetic coronal image resembles a blurred coronal image. This fact opens the possibility of adjusting the model empirically based on the laboratory measurements and calibrations. Additionally, it may allow the development of a simplified algorithm for the ghost calculation.

We showed that the ghost image can be calculated based on the image recorded at the detector, that is, an image that contains the ghost signal. The removal procedure works very efficiently, and after the removal, the relative contribution of residual ghost light amounts to $10^{-5}$ of the local coronal signal. The procedure yields good results even with a stronger contribution of ghosts, for example with the removed detector glass and the ND50\% filter (see Appendix B). This fact raises the question of the necessity of the detector glass and the ND50\% filter, as, besides reducing the ghost contributions, the glass introduces its own ghosts. Any possible tilt of the glass (even very small) may further complicate the situation.

For a comparison with other stray light sources, we took the diffraction calculated from Rougeot et al. (2017), and Shestov \& Zhukov (2018), and ABg scattering on the lens surfaces. To model the scattering, we took the worst-case $\mathrm{ABg}$ parameters with TIS $=3 \times 10^{-4}$ and studied individually the effect on the detector produced by every lens. The main contributors are the $\mathrm{O} 2$ and $\mathrm{O} 3 / \mathrm{L} 1$ lenses. We calculated the scattering of coronal and diffracted light due to these lenses. We also analyzed scattering of the intense incoming diffracted light (whose total intensity is 3 orders of magnitude larger than the intensity coming to the detector) by the primary objective without occultation by the IO. We believe this approach is sufficient to obtain a qualitative result.

The analysis shows that the major contributor to the stray light is diffraction. Its intensity is at least one order of magni- tude larger than ghost light or scattering, and amounts to $10 \%$ of the observed coronal signal in the outer corona region. The intensity of the ghost light is almost ten times smaller in the outer region, and the intensity of the scattered light (calculated in the raytracing/BSDF approach) is smaller than the intensity of the ghost light. The small contribution of the ghost light in comparison to diffraction adds further weight to the argument for the non-necessity of the detector glass. The use of the detector glass inevitably introduces an additional ghost component, and use of the ND50\% reduces the total throughput of the telescope. This may be especially important for observations of polarized light. Recent consideration of diffraction and scattering together (Rougeot et al. 2018b) shows that, due to scattering, the diffracted light increases its intensity in the outer corona (this is not taken into account in other models). This possibility even further increases the importance of the diffracted light and reduces relative contributions of other sources.

We showed that the ghost light produced by the primary objective is relatively weak due to ASPIICS's optical design. Therefore, we conclude that the Lyot spot (used in LASCO C2 and LASCO C3) is not necessary in ASPIICS.

The unique feature of the ASPIICS coronagraph provided by the FF capability is the observation of extremely low corona, starting from heights as low as $\sim 1.08 R_{\odot}$. Such observations are essentially impossible to obtain with other types of externally occulted space coronagraphs because of significant stray light and vignetting. In fact, the requirements to reduce stray light and simultaneously minimize the inner observational height effectively counteract each other, as the diffraction in the internal zone decreases only with the increase of the occultation and vignetting of the corona (Rougeot et al. 2017). Additional stray light on the detector not only increases possible noise in each individual pixel due to photon noise, but more importantly alters the inferred photometry. Photon noise (as well other types of noise) can be reduced by using two sequential exposures or averaging over several pixels, whereas systematic overestimation of measured intensity due to stray light may result in misinterpretation of observational data. Careful laboratory measurements of the ghost light and scattered light performance are necessary for the development of successful on-ground data processing. An especially important role is played by validation of the correctness of the adopted models, that is, confirming the absence of other sources of stray light, correct identification of the major ghost sources, the applied scattering model, and so on.

Current models for the stray light, which take into account diffraction (Rougeot et al. 2017; Shestov \& Zhukov 2018), ghost reflections (the present analysis and Galy et al. 2018), and scattering (Rougeot et al. 2018b), show that the total contribution of the stray light will be less than $10 \%$ of the coronal signal in almost the whole FOV. This makes the ASPIICS an instrument that will provide unique observations of the low solar corona.

Acknowledgements. S. V. S. and A. N. Z thank the European Space Agency (ESA) and the Belgian Federal Science Policy Office (BELSPO) for their support in the framework of the PRODEX Programme.

\section{References}

Allen, C. W. 1976, Astrophysical Quantities, 3rd edn. (Athlon)

Bayanna, A. R., Mathew, S. K., Sankarasubramanian, K., et al. 2011, Exp. Astron., 29, 145

Bernaerts, D., Bermyn, J., \& Teston, F. 2002, in PROBA (Project for Onboard Autonomy), eds. M. Rycroft, \& N. Crosby (Dordrecht, Netherlands: Springer), 53

Bout, M., Lamy, P., Maucherat, A., Colin, C., \& Llebaria, A. 2000, Appl. Opt., 39,3955 
Brueckner, G. E., Howard, R. A., Koomen, M. J., \& Korendyke, C. M. 1995, Sol. Phys., 162, 357

Dolla, L., \& Solomon, J. 2008, A\&A, 483, 271

Eyles, C. J., Harrison, R. A., Davis, C. J., et al. 2009, Sol. Phys., 254, 387

Galano, D., Buckley, S., Cernica, I., et al. 2018, in Space Telescopes and Instrumentation 2018: Optical, Infrared, and Millimeter Wave, eds. H. A. MacEwen, M. Lystrup, G. G. Fazio, et al. Proc. SPIE, 10698, 106982Y

Galy, C., Fineschi, S., Galano, D., et al. 2015, in Solar Physics and Space Weather Instrumentation VI, Proc. SPIE, 9604, 96040B

Galy, C., Thizy, C., Stockman, Y., et al. 2018, in Internal Conference on Space Optics (ICSO) 2018, ed. N. Kadowaki (SPIE), 52

Goryaev, F., Slemzin, V., Vainshtein, L., \& Williams, D. R. 2014, ApJ, 781, 100

Hahn, M., Landi, E., \& Savin, D. W. 2011, ApJ, 736, 101

Halain, J. P., Eyles, C. J., Mazzoli, A., et al. 2011, Sol. Phys., 271, 197

Harvey, J. E., Choi, N., Schroeder, S., \& Duparré, A. 2012, Opt. Eng., 51, 51

Högbom, J. A. 1974, A\&AS, 15, 417

Kim, I. S. 1997, in NATO Advanced Science Institutes (ASI) Series C, eds. Z. Mouradian, \& M. Stavinschi, 494, 159

Koutchmy, S. 1988, Space Sci. Rev., 47, 95

Lamy, P., Damé, L., Vivès, S., \& Zhukov, A. 2010, in Space Telescopes and Instrumentation 2010: Optical, Infrared, and Millimeter Wave, Proc. SPIE 7731,773118

Landini, F., Mazzoli, A., Venet, M., et al. 2010, in Ground-based and Airborne Instrumentation for Astronomy III, Proc. SPIE, 7735, 77354D

Landini, F., Vives, S., Romoli, M., et al. 2017, in International Conference on Space Optics - ISCO 2012, Proc. SPIE, 10564, 105640F

Leyre, X., Sghedoni, M., Vives, S., Lamy, P., \& Pailharey, E. 2005, in UV/Optical/IR Space Telescopes: Innovative Technologies and Concepts II, ed. H. A. MacEwen, Proc. SPIE, 5899, 221
Llebaria, A., Lamy, P. L., \& Bout, M. V. 2004, in Telescopes and Instrumentation for Solar Astrophysics, eds. S. Fineschi, \& M. A. Gummin, Proc. SPIE, 33, 5171

Llebaria, A., Loirat, J., \& Lamy, P. 2012, in Space Telescopes and Instrumentation 2012: Optical, Infrared, and Millimeter Wave, Proc. SPIE, 8442,844226

Pfisterer, R. N. 2011, Opt. Eng., 16

Renotte, E., Alia, A., Bemporad, A., et al. 2015, in Solar Physics and Space Weather Instrumentation VI, Proc. SPIE, 9604, 96040A

Rougeot, R., \& Aime, C. 2018, A\&A, 612, A80

Rougeot, R., Flamary, R., Galano, D., \& Aime, C. 2017, A\&A, 599, A2

Rougeot, R., Galano, D., Kirschner, V., et al. 2018a, in Space Telescopes and Instrumentation 2018: Optical, Infrared, and Millimeter Wave, eds. H. A. MacEwen, M. Lystrup, G. G. Fazio, et al., Proc. SPIE, 10698, 106982T

Rougeot, R., Flamary, R., Mary, D., \& Aime, C. 2018b, A\&A, submitted

Sandri, P., Naughton, D., Sandri, P., et al. 2018, Opt. Eng., 57, 1

Seaton, D. B. 2001, Senior Honors Thesis (Williamstown, Massachusetts: Williams College)

Shearer, P., Frazin, R. A., Hero, III., A. O., \& Gilbert, A. C. 2012, ApJ, 749, L8

Shestov, S. V., \& Zhukov, A. N. 2018, A\&A, 612, A82

Thompson, W. T., Davila, J. M., Fisher, R. R., et al. 2003, in Proc. SPIE, eds. S. L. Keil, \& S. V. Avakyan, 4853, 1

Thompson, W. T., Wei, K., Burkepile, J. T., Davila, J. M., \& St. Cyr, O. C., 2010, Sol. Phys., 262, 213

Vivès, S., Lamy, P., Levacher, P., Boit, J. L., \& Saisse, M. 2006, in Society of Photo-Optical Instrumentation Engineers (SPIE) Conference Series, Proc SPIE, 6265, 626524

Wang, T., Reginald, N. L., Davila, J. M., St. Cyr, O. C., \& Thompson, W. T., 2017, Sol. Phys., 292, 97

Weliachew, L., Emerson, D. T., Forveille, T., et al. 1985, A\&A, 153, 139

Wendeln, C., \& Landi, E. 2018, ApJ, 856, 28 


\section{Appendix A: Raytracing in Zemax OpticStudio}

In order to analyze properties of ghost images, we use a raytracing software package - Zemax OpticStudio ${ }^{1}$ in various regimes. In a typical user scenario the user sets up the optical layout by specifying the relative positions, radii of curvatures, thicknesses, and diameters of the lenses, as well as glass material and possibly other properties (e.g., coating). The user also provides the input beam (one or several) by specifying its field angle. Then a set of rays are launched by Zemax. The rays have the same field angle but are shifted with respect to each other perpendicularly to the optical axis to fill the whole entrance aperture of the optical system. Zemax traces every ray, calculating (within numerical accuracy) the coordinates of the intersection of the ray with a particular lens' surface, taking into account all geometrical factors. Then the angles of incidence and transmission are calculated, and the ray is traced further until the next surface and so on, until it ultimately reaches the detector. The final position of all the incoming rays produces the point spread function (PSF) of the telescope. This regime of the Zemax operation is called sequential, because every individual ray passes all the lenses in the order of their $z$-coordinate and never gets split.

In sequential analysis, Zemax also provides the user with the first-order parameters of the optical system, such as effective focal length $f$, working $f$ /\# number, magnification, etc. These parameters are obtained in the paraxial regime (i.e., limit of very small ray angles and heights), however these parameters are not applicable when the full geometry model (not paraxial) is used, that is, not applicable already in the sequential regime.

In its nonsequential regime, Zemax allows every individual ray to be split on a lens surface, giving rise to additional ghosts or scattered rays. The newly introduced rays (their actual number depends on many factors) carry a small amount of intensity of the mother ray in accordance with reflective or scattering properties of the surface. Afterwards all the rays are considered in a similar manner and are raytraced further, however the information about every ray origin is preserved and can be used afterwards. In this regime no rule of sequential propagation through lenses is applied. Since during consideration of ghost light and scattering the number of new rays increases as an avalanche with the increase of number of optical surfaces, and their intensity decreases dramatically with the number of backrefletions/scattering events, various limitations (like minimal intensity or maximal number of splits) are used to keep the number of rays under analysis reasonable. In particular, we had to fine-tune some parameters of Zemax to be able to reveal all the ghost rays (from all the surfaces) on the detector. During the investigation of scattering we were able to consider scattering on lenses individually, that is, initially first lens, then second lens, and so on. Turning on scattering on all the surfaces gave nonphysical results, as the scattered rays from the former surfaces were omitted.

There exists a possibility of analyzing ghost images in Zemax in paraxial regime. We did not find an exact explanation of the feature in the Zemax documentation, but we believe it is done as follows: to investigate the ghosts formed by two particular surfaces, Zemax automatically duplicates the given part of the optical system and considers paraxial ray propagation within the modified optical layout. Within the paraxial approximation such parameters as effective focal length, position of exit pupil, effective $f$ /\# ratio, and so on are calculated, however they are valid only for the modified system, i.e., for a particular ghost image.

\section{Appendix B: Ghost light with no detector glass}

In this section we analyze the behavior of the ghost light in cases where we completely remove the detector glass from the optical system. Here, we take the synthetic coronal scene as in Sect. 5.2 and modify the Fortran code in order to completely remove the contribution of the detector glass (corresponding ghosts) and ND50\% filter (decrease of intensity of the remaining ghosts). In Fig. B.1 we present a comparison of the observed image $\mathbf{R}$ (panel a), ghost image $\mathbf{G}(\mathbf{R})$ (panel b) and the difference $\mathbf{I}-\mathbf{C}$ after the removal procedure (panel c). The color scale is normalized to the recorded image, however the intensity in panel (a) is divided by ten for visualization purposes. The dynamic range of the color scale is modified with respect to Figs. 9 and 10 in order to reveal weaker intensities. In the bottom panel, we plot horizontal profiles of the registered image (black line; factor 0.1), ghost image (blue line) and the difference (red line; factor 10).

Removing the detector glass causes the relative intensity of ghosts to increases by a factor of approximately four as the ND50\% filter is no longer present to reduce ghost contributions. In this case, the intensity of the ghosts amounts to $10 \%$ of the corona at heights $>2.2 R_{\odot}$. However, after the removal procedure the remaining signal has a relative amplitude of $<10^{-4}$ and has a more uniform structure. We attribute this to the modified structure of the ghost image, as the bright and relatively contrasted contributions from the detector glass are absent.

1 https://wwW.zemax.com/products/opticstudio 
A\&A 622, A101 (2019)
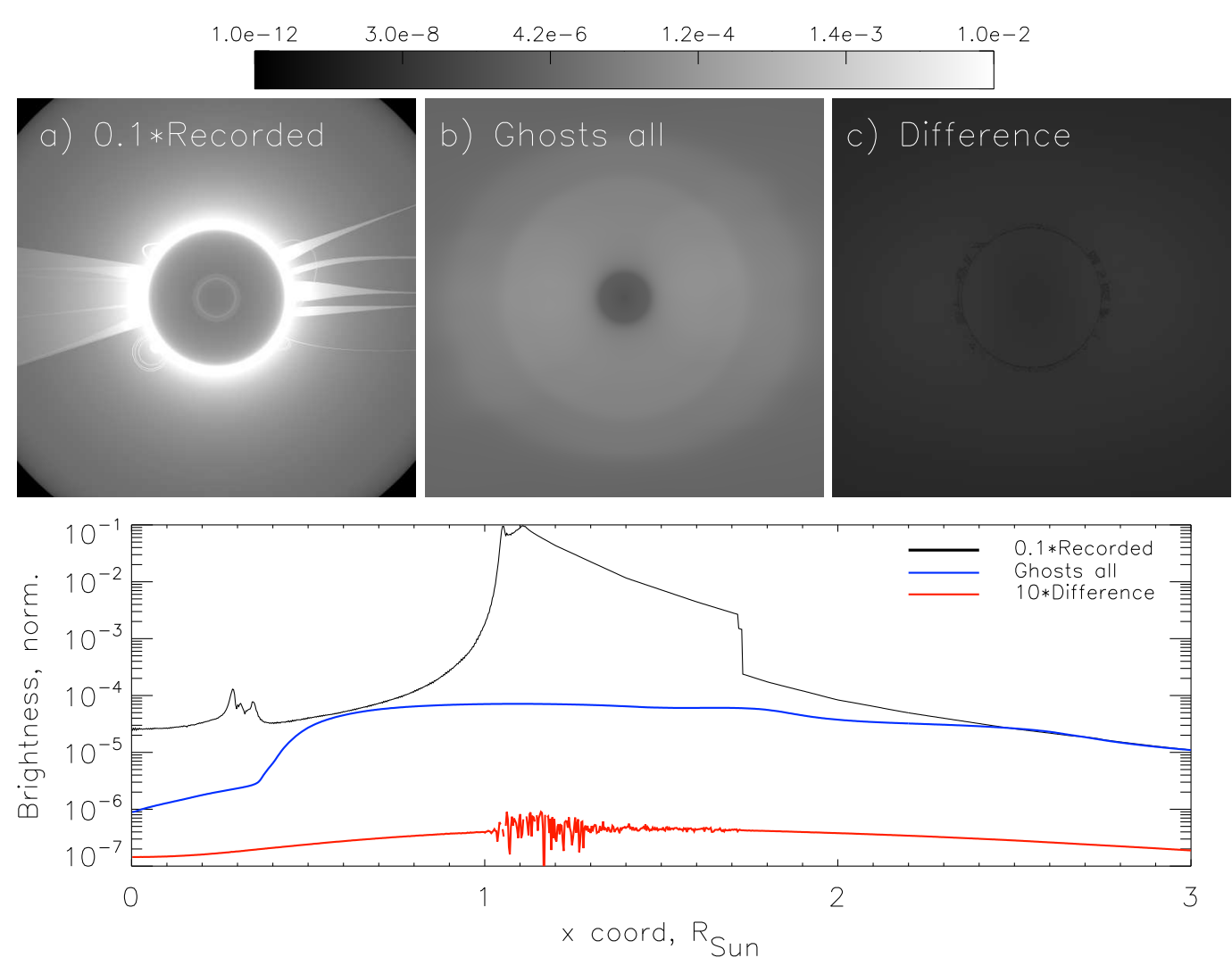

Fig. B.1. Analysis of the removal procedure for the synthetic coronal image with the removed detector glass. Top panels: same notation as in Fig. 9, bottom panel: intensities of $\mathbf{R}, \mathbf{G}(\mathbf{R})$ and $\mathbf{I}-\mathbf{C}$ in the westward from the disk center direction. 